2,179 , in the fall courses within the quadrangles, and a considerable loss, from 562 to 247 , in the courses outside the quadrangles. Owing to the increase in the summer session figures, however, there is a gain in the grand total. The change of courses given for teachers from the center of the city to the quadrangles, which took effect this fall, has lessened the number of students' in such courses, but increased the efficiency of the work.

As for individual schools, there has been a gain in the academic department, especially in men, in law and in pedagogy, while there has been a slight loss in medicine, divinity and the graduate schools. As in the case of the University of Pennsylvania, a number of students enrolled in courses for teachers have been included in the Chicago figures who would be excluded in the Columbia or Harvard figures, but the time for making more definite inquiries was too short.

The following errors should also be noted: In the list of institutions mentioned on page 794, column two, line eleven, Stanford should be inserted between Kansas and Indiana; and in line fifteen Chicago should be omitted. On page 796, column one, line eighteen, Chicago should be inserted before Harvard. In the table, the number of men in the academic department of Princeton University should be 758, instead of 755. On page 794, column one, line twenty, insert, before Missouri, 'Syracuse (48.71\%).' Rudolf Tombo, JR.

\section{ALCOHOL FROM CACTI}

To THE Editor of ScIEnce: In a letter entitled 'Alcohol from Cacti,' which appeared in the Scientific American for December 15, the author referring to the results obtained with this plant by a California chemist, states that "from five pounds of pulp he distilled, in a crude way, more than a gallon of alcohol, which was clear in color, and burned readily with a bright, warm glow."

At the time this article appeared we were hesitating about publishing the enclosed press bulletin for fear the theoretical estimates therein given would exceed the amount which it would be possible to obtain in practise.

Cactus will not average over 10 per cent. carbohydrates, and if, as is usually estimated, this yields one half its weight of 95 per cent. alcohol, it is not clear how it would be possible to obtain one gallon of alcohol from less than
140 pounds of this plant. If, however, the chemist referred to above can distil one gallon (seven pounds) from five pounds of cactus pulp, it would be interesting to know what the strength of his product is, and whether or not it was done with the assistance of a magician's wand.

\section{Agricultural College, NeW MeXICo}

\section{THE PARTHENOGENESIS OF ENCYRTUS}

AT the time that my recent note on 'Polyembryony and Sex-determination' was written I had not seen Silvestri's latest communication. In a brief, preliminary paper ${ }^{1}$ he presents the results of his studies on the early stages of the development of Encyrtus and among other details notes the fact that, as in Litomastix, there is parthenogenetic development, unfertilized eggs always producing males, fertilized ones only females. The maturation and early segmentation stages studied are identical in the two types.

\section{WM. A. RILEY}

\section{SPECIAL ARTICLES}

POLARIZATION AND INTERFERENCE PHENOMENA WITH WHITE LIGHT

I HAVE usually found great difficulty in endeavoring to explain the color phenomena obtained with white light in rotary polarization, in the behavior of thin plates with or without polarized light, and in interferences and diffractions generally, to an elementary class. The following diagram, therefore, which yields a large amount of information, may be of interest to the reader, although it contains nothing essentially novel. Note the occurrence of $d / \lambda$ throughout.

Rotary Polarization.-If we write the rotation $\theta$ of the plane of polarization due to a thickness $d$ of quartz cut perpendicularly to the axis,

$$
\theta=\pi\left(1-v^{\prime} / v^{\prime \prime}\right) \cdot d / \lambda^{\prime},
$$

where $v^{\prime}$ and $v^{\prime \prime}$ are the velocities of righthanded and left-handed rays in the crystal

${ }^{1}$ Silvestri, F., 1906, 'Sviluppo dell Ageniaspis (Encyrtus) fuscicollis (Dalm.) Thoms.,' Atti Acc. Lincei (5), XV., pp. 650-658. 


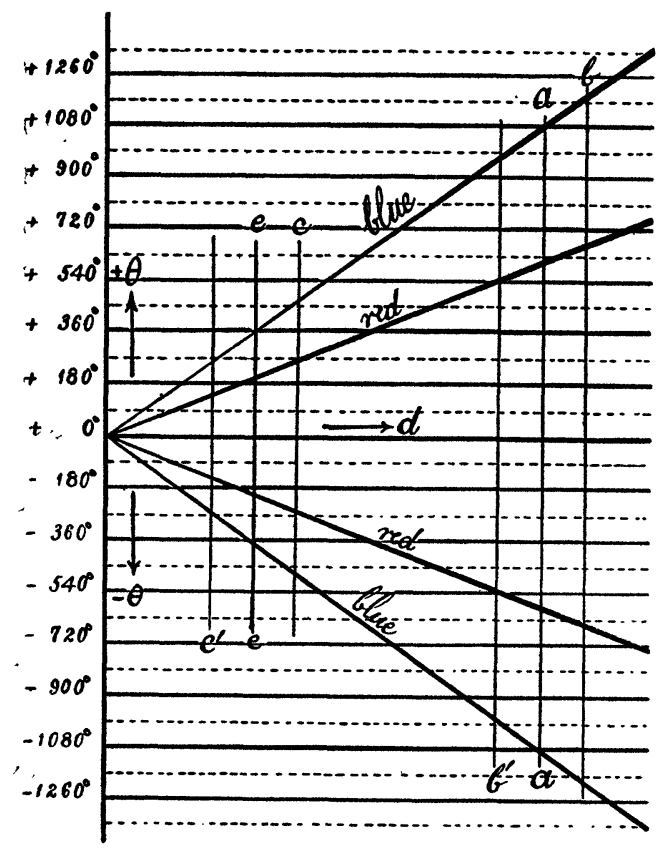

and $\lambda^{\prime}$ the wave-length or pitch of the righthanded screw, it is convenient to lay off $\theta$ in terms of $d$ as in the chart. Two oblique lines in the positive field may then represent the amount of rotation corresponding to any two typical colors, as for instance, blue and red. The former having the smaller wave length will have the steeper slope. If the rotation is left handed, two other symmetrically oblique lines below the axis will represent this case. If the nicols are crossed the colors which fail to get through the analyzer must be rotated in multiples of $\pi$. Hence horizontal black bars intersect the whole field above and below the abscissa, to point out the colors blotted out. Colors which are rotated in odd multiples of $\pi / 2$ get completely through, and these are shown by the dotted horizontal bars midway between the former. If the nicols are in parallel the black and dotted bars replace each other; or in general, if the analyzer is rotated $\theta$ to the right or to the left, the whole system of bars will move up or down, respectively, by the amount $\theta$, as the dark band will pass through the spectrum of the right-handed plate from red to blue. The colors to be anticipated for any thickness of plate will then be given by drawing vertical lines as $a, b, c$, etc. Thus it is clear that only for relatively thin plates may vivid colors be expected, for here (as at $c$ and $e$ ) there is but one or a few extinction bands in the spectrum. As the thickness increases the number of these bands increases until eventually the colors removed are practically the same as those retained and white light appears in all positions of the nicols. The spectra are channeled in the beautiful way seen with a column of quartz an inch or more long.

Furthermore, if a right and left bi-quartz be taken as in the saccharimeter the colors for the same $d$ will be identical both for crossed and parallel nicols, but not otherwise. For the dark bands move from red to blue and from blue to red, respectively, in the two halves, on like rotation of the nicols. Moreover, if for a thickness $d$ an additional rotation is imparted as by the sugar tube, $d$ will pass to $c$ in the right-handed plate but to $c^{\prime}$ in the left-handed plate. In relatively thin plates, however, the identity of color may be restored on rotating the analyzer to the right, for the dark band at $c^{\prime}$ below the abscissa moves in wave-lengths much more rapidly through the spectrum than at $c$ above the abscissa; but the identical colors will even here not be quite the original color. For a relatively great thickness the identity can not be restored since $b$ contains three and $b^{\prime}$ usually two extinction bands.

Finally the occurrence of successive orders of colors is suggested by the chart.

Thin Plates in Polarized Light.-The plates of thickness $d$ are cut parallel to the axis, or the edge of the thin wedge is so ground and placed at 45 degrees to the plane of the polarizer. If we write

$$
\phi=2 \pi\left(\mu_{e}-\mu_{o}\right) \cdot d / \lambda,
$$

where $\phi$ is the phase difference of ordinary and extraordinary rays, $\lambda$ the wave-length in air, $\mu_{0}$ and $\mu_{e}$ the respective indices of refraction, and if the nicols are crossed, the diagram as drawn will apply at once. Rotation of the analyzer over 90 degrees exchanges the black and dotted horizontal bars as before; but there is this essential difference, that the bars can not be moved continuously on rotating 
the nicols, since the intermediate colors are elliptically polarized. Colors are white for thick plates and one may note in addition that $\mu_{e}-\mu_{o}$ and $d$ are reciprocally related.

Interference. Diffraction.-For the case of two slits, real or virtual, at a distance $c$ apart and $r$ from the screen, if $d$ is the distance of a lateral dark band from the central fringe for light of wave length $\lambda$, we may write

$$
n=(2 c / r) \cdot d / \lambda
$$

where odd numbers for $n$ determine the position of the successive minima. Hence if the phase difference in which the rays meet $\phi=\pi n$ be introduced,

$$
\phi=(2 \pi c / r) \cdot d / \lambda
$$

and the chart is applicable at once, with the understanding, however, that the dark horizontal bars now denote maxima, the dotted bars minima. This, however, is not necessary, for the bars may be moved up by inserting in one of the rays a thickness of lamina $t$ of the refracted index $\mu$ (compensator) by the amount $2 \pi(\mu-1) t / \lambda$, until a minimum replaces the central maximum. The compensator thus has the same effect as the rotation of the nicols in the first paragraph. The chart shows the lateral extent of spectra of different orders very clearly, the first having a breadth of $d=0$ the other breadths being proportional to the intercepts of the successive bars between the oblique lines blue and red. The overlapping of these spectra is also well shown. The spectra must be less spread out as the slopes $2 \pi c / r \lambda$ are steeper. If the slit of the spectroscope be placed at a given distance, as at $d=a$, for instance, the vertical section indicates the channeled spectra which will be observed and the dark lines may be sharp enough to suffice the standardizing the spectrum.

With certain well-known changes the same remarks apply for most cases of the diffraction of white light.

Colors of Thin Films. Ordinary Light.-If $d$ denote the thickness of the film or a given section of the wedge of refractive index $\mu, \lambda$ the wave length of light in air, $r$ the angle of refraction corresponding to the angle of incidence $i$ we may write

$$
\phi=2 \pi \mu \cos r \cdot d / \lambda
$$

where minima correspond to even numbers of $\pi$. Hence the positive field of the chart applies at once. The equation as written is primarily useful for the wedge. What the diagram points out very well is the resolution of doublets in the spectra of very high order. If the incidence is normal and the wedge be of air as in the interferometer $\phi=2 \pi d / \lambda$. If the thickness is constant and the angle of incidence varies the oblique lines are still applicable if instead of $d$ the quantity $\cos r$ be laid off as the abscissa. They are real between $r=0$ and the angle of total reflection.

In practice the oblique lines may be drawn to the scale on a sheet of paper, the black bars on a sheet of celluloid capable of sliding up and down over the former and the vertical lines may be represented by threads movable to right and left over the celluloid. The whole is to be serviceably framed on a sheet of tin plate.

\section{Brown UNIVERsity, Providence, R. I.}

\section{THE CAUSES OF THE GLACIAL EPOCH ${ }^{2}$}

OF the various hypotheses advanced to account for the oceurrence of a protracted glaciation of a large portion of the earth's surface in post-tertiary times, none has thus far met with universal acceptance; partly because of the insufficiency of data regarding critical geographical areas, but mainly because of the feeling that although the causes discussed by Croll, Chamberlin and others are or may be true ones, they are inadequate to account, quantitatively, for all the facts observed. The paleontological evidence of the prevalence of temperate and even semi-tropic floras and faunas in the late Tertiaries within what are now arctic regions, gives such forcible evidence of the comparative uniformity of tem-

${ }^{1}$ Read at tenth session of the International Geological Congress, Mexico, September 6-14, 1906. 